\title{
Efficacy of acupuncture in asthma
}

\section{To the Editor:}

I read with interest the recent meta-analysis by MARTIN et al. [1], finding little evidence for an effect of acupuncture as a treatment for asthma, but was not surprised by the result. I spent my 6-week medical student elective in 1988 at the ("Western"-style medicine) Guangzhou Institute of Respiratory Diseases, Guangzhou, China. As part of the elective I reviewed, with the help of the clinic doctors, any treatments currently being used by 58 of 66 asthmatic patients attending the weekly respiratory clinic. While all except a first-time patient were taking Western treatments prescribed at the clinic, $50(86 \%)$ had seen a traditional Chinese medical doctor for their asthma and $32(55 \%)$ were currently using Chinese herbs. Lists of herbs being used were provided by 17 patients and 15 of these included ma huang (herba ephedra), the plant from which ephedrine was first isolated. However, only 18 $(31 \%)$ patients had ever tried acupuncture for their asthma, five of whom volunteered that acupuncture had little effect. Four of the patients had had acupuncture on one of the san fu tian, the three hottest days of the year according to the lunar calendar, to reinforce the body's yang to protect against winter asthma in the yin time of year. I was also told that in some areas, injection with dead tubercle into acupuncture points was used as a treatment for patients with intrinsic asthma. This is interesting in view of the recent discussion relating to the role of bacilli Calmette-Guérin vaccination and mycobacteria in relation to allergic disease development [2].
Asthmatic patients choosing to attend a Western-style hospital may have been a selected group not responding to acupuncture. However, doctors at the Guangzhou Traditional Chinese Medical College, which I also visited during my elective, held the opinion that acupuncture was not the treatment of choice for asthma as it was not particularly effective. MARTIN et al. [1] conclude from their meta-analysis that a full-scale randomised clinical trial is needed, but my experiences lead me to suggest that some prior qualitative work with Chinese medical practitioners treating asthmatic patients is needed to define the parameters of such a study, if one is in fact needed.

\section{A. Hansell}

Dept Epidemiology and Public Health, Imperial College, London, UK.

\section{References}

1. Martin J, Donaldson ANA, Villarroel R, Parmar MKB, Ernst E, Higginson IJ. Efficacy of acupuncture in asthma: systematic review and meta-analysis of published data from 11 randomised controlled trials. Eur Respir J 2002; 20: 846852.

2. Strachan DP. Family size, infection and atopy: the first decade of the "hygiene hypothesis". Thorax 2000; 55: Suppl. 1, S2-S10.

\section{Asthma variability}

\section{To the Editor:}

The article by ZHANG et al. [1] demonstrated the ability to reanalyse previously published data and reformulate the conclusions. The analysis describes the hetereogeniety of asthma-treatment response among patients treated with inhaled corticosteroids, montelukast or placebo. Although these studies demonstrated that both active therapies are superior to placebo and that beclomethasone is superior to montelukast, appropriately, the authors point out that the response to specific end-points is inconsistent. They illustrate the response to active treatment quite well with distribution curves and conclude that there is no simple haplotype to describe a responder. They propose that response to therapy is dependent on multiple variables. There is a normal unimodal distribution curve for lung function and symptom scores for both active treatments as well as placebo. The active therapies show the distribution curve moving in the direction of improvement, with beclomethasone greater than montelukast. Studying the placebo response illustrates the variable nature of asthma, as some placebo-treated patients exhibit significant improvement, some significant worsening and many remain unchanged. Beyond studying the impact of the medication is the importance of understanding the variable nature of asthma, which is essential in understanding the true response or lack of improvement following therapy. The logic of finding a genetic response would also suggest that if one builds a model for studying pharmacogenomic haplotypes for active therapies, one must also consider a model to study placebo haplotypes.

The "within-patient variability" probably reflects the unpredictability of the disease. The definition of asthma of the National Heart, Lung and Blood Institute (NHLBI) [2] includes "widespread but variable airflow obstruction that is often reversible either spontaneously or with treatment." This variable nature of the disease may be more important than finding the "surrogate clinical marker of asthma control." Mean data may be relevant and studying multiple outcomes is essential. When the majority of end-points demonstrate that one therapy is better than the other, then the search for the ultimate end-point may be unnecessary. Although there is not a clear relationship between the end-points that are measured, what is unambiguous is that inhaled corticosteroids for all clinical end-points are superior to leukotriene-receptor anatagonists. This article is a good intellectual exercise but does not change previous conclusions. The ultimate end-point 
in evaluating asthma is to achieve the goals of asthma care: reduce symptoms and exacerbations. NHLBI [2] and the Cochrane Library Database [3] have used formal evidencebased criteria to review the literature and have concluded that inhaled corticosteroids are the preferred initial controller therapy for persistent asthma.

Rather than trying to re-evaluate data, new studies should be designed with alternative therapies that provide greater improvement in lung function, better symptom control and fewer exacerbations, with the understanding that the differences in response should also take into consideration the variable nature of the asthma.

\section{D.A. Stempel}

Dept of Pediatrics, University of Washington, Bellevue, WA, USA.

\section{References}

1. Zhang J, Yu C, Holgate ST, Reiss TF. Variability and lack of predictive ability of asthma and end-points in clinical trials. Eur Respir J 2002; 20: 1102-1109.

2. National Asthma Education and Prevention Program, Expert Panel Report: Guidelines for the diagnosis and management of asthma update on selected topics - 2002. J Allergy Clin Immunol 2002; 110: s141-s219.

3. Ducharme FM, Hicks GC. Anti-leukotriene agents compared to inhaled corticosteroids in the management of recurrent and or chronic asthma in adults and children. Cochrane Database of Systemic Reviews, Issue 3, 2002.

\section{From the authors:}

We thank D.A. Stempel, for the opportunity to restate and clarify the dialogue we had hoped to initiate with our paper [1]. As described in our paper, the primary efficacy results from these trials have previously been published. The message of our present paper, different from that of the letter by D.A. Stempel, is therefore not one of comparisons of drug treatments. In fact, there was no presentation or discussion of such data in our paper. Instead, we have engaged in an activity that he dismisses as an "intellectual exercise", with the hope of spurring further research and debate in the critical area of understanding asthma control.
The rationale for our work was to provide empirical evidence of the known variability of asthma as reflected by the key measures describing the disease process. In this context, we point out the inherent difficulties in studying this disease, particularly when relating it to haplotype associations. As D.A. Stempel notes, the aim of asthma treatment is control of the disease. Efforts to define and accurately validate the important clinical measures of this goal have provoked much debate, and uncertainty remains about which outcome or combination of outcomes in asthma will define and serve to measure control of this disease (just as fracture risk has been identified in osteoporosis, for instance). Unlike our appreciation of the relationship between bone mineral density and fracture risk in osteoporosis, or blood pressure and stroke risk in hypertension, we have a limited understanding of the clinical relevance of the measured surrogate markers (e.g. forced expiratory volume in one second, wheezing) in asthma.

The variable nature of asthma, which is the focus of our paper, is a confounding factor that makes the task of understanding how to control the disease all the more difficult. Our results show that commonly used clinical endpoints measure different aspects of the disease state. These end-points may not capture adequate information to serve as predictors of long-term response to therapy. Our paper clearly points to the urgent need to frame the debate about asthma control in terms of clinically relevant outcomes, and the response to treatment in terms of relevant clinical markers.

D.A. Stempel's simple view of the evidence as reflected by published mean values that are summarised in meta-analyses does not begin to account for the complexity of measuring and understanding asthma control.

\section{J. Zhang*, C. Yu*, S.T. Holgate" ${ }^{\#}$ T.F. Reiss}

Depts of ${ }^{*}$ Clinical Biostatistics and ${ }^{\circ}$ Clinical Research, Merck Research Laboratories, Rahway, NJ, USA. "Respiratory, Cell and Molecular Biology Research Division, School of Medicine, University of Southampton, Southampton, UK.

\section{References}

1. Zhang J, Yu C, Holgate ST, Reiss TF. Variability and lack of predictive ability of asthma end-points in clinical trials. Eur Respir J 2002; 20: 1102-1109. 